\title{
Assessing the quality of the relation between scalarizing function parameters and solutions in multiobjective optimization
}

\author{
José C. Ferreira, Carlos M. Fonseca, and António Gaspar-Cunha
}

\begin{abstract}
Different Multi-Objective Optimization Methods (MOOM) for solving Multi-Objective Optimization Problems (MOOP) have been suggested in the literature. These methods often comprise two stages (not necessarily sequential): i) the search for the Pareto-optimal set and ii) the selection of a single solution from this non-dominated set. Various studies comparing performance of particular aspects of these methods have been carried out. However, a theoretical support that changes on the preferences of a Decision Maker (DM) will be reflected in the same way on the solution of the MOOP given by the MOOM has not been presented. In this work a consistency measure to assess MOOM is proposed. It will used to compare the performance of different methods available in the literature. This study was performed using some benchmark test problems, with two criteria.
\end{abstract}

\section{INTRODUCTION}

$\mathrm{R}$ EAL world optimization problems are characterized by the existence of multiple, often conflicting, objectives (or criteria) to be satisfied simultaneously. In the absence of information about the relative importance of the various criteria, such problems typically admit multiple optimal solutions where any improvement in one objective can only be obtained at the expenses of degradation in another, i.e., the Pareto frontier [1-3]. Thus, in order to distinguish the preferred solutions among the non-dominated ones, a Decision Maker (DM) must be able to articulate a preference structure between the various criteria. This means that solving a multi-objective optimization problem consists, not only of obtaining the Pareto frontier, but also of selecting a single solution from this set. Several methods for solving complete multi-objective optimization problems, i.e., to obtain a single preferred solution, have been developed in the last years [4].

Depending on how the search and decision processes are interconnected, three different classes of multi-objective preference methods can be identified [4], [5]:

A priori methods: The decision maker must specify her or

Manuscript received October 12, 2008. This work was supported in part by the Portuguese Fundação para a Ciência e Tecnologia under grants: PTDC/GES/70168/2006 and SFRH/BD/22808/2005.

J. C. Ferreira is with the IPC/I3N Institute for Polymers and Composites, University of Minho, Campus de Azurém, 4800 Guimarães, Portugal (email: jcf@dep.uminho.pt).

C. M. Fonseca is with the Faculty of Science and Technology, Universidade do Algarve, Campus de Gambelas, 8005-139 Faro, Portugal (e-mail: cmfonsec@ualg.pt), and CEG-IST, Instituto Superior Técnico, Universidade Técnica de Lisboa, Portugal.

A. Gaspar-Cunha is with the IPC/I3N Institute for Polymers and Composites, University of Minho, Campus de Azurém, 4800 Guimarães, Portugal (corresponding author: phone: +351253510328; e-mail: agc@ dep.uminho.pt). his preferences, expectations and/or options before the optimization process takes place. The preferences are expressed in terms of an aggregating function which combines individual criterion values into a single utility value.

A posteriori methods: After the generation of the Pareto optimal set, the DM selects the most preferred among the alternatives taking into account his or her own preferences.

Interactive methods: Decision making and optimization occur at interleaved steps. At each step, partial preference information is supplied by the DM to the optimizer, which, in turn, generates better alternatives according to the information received.

Recently, an approach based on a combination of a-priori and a-posteriori methods was proposed [6].

Various studies comparing the performance of these methods have been carried out [7-11]. They concluded that their performance depends on the type of measures used for comparison. These studies addressed, mainly, linear problems which are solved using interactive methods. Usually, the performance assessment is made using either a human DM or value functions [12] to replace the DM. Both types of testing methods are important, depending on the motivation for the evaluation [13]. The practical applicability of a method, such as ease of use and confidence on the solution obtained, must be tested using a human DM, whereas theoretical performance, such as the rate of convergence, CPU time, and number of Pareto optimal solutions needed to solve the problem, must be tested using value functions [13].

The usability of interactive methods depends strongly on the extent to which the parameter values set by the DM as an expression of his or her preferences lead to solutions corresponding to those preferences. For example, it is well known that solutions obtained by combining objectives using the weighted-sum approach often do not correspond to the preferences the DM wished to express when setting the weights, to the point where weights are seen as no more than "technical" parameters. As a consequence, iterative tuning of the weights is usually required until a satisfactory solution is obtained.

In this work, the quality of the relation between decisionmodel parameters and the corresponding solutions is of interest. Ideally, the objective values of the non-dominated solution selected should reflect, in a consistent way, the changes made to the parameter vector. Therefore, a suitable quality measure is proposed, and a comparative study is performed involving two classical preference articulation 
methods (Weighted Metrics and Goal Programming) as well as a third method, recently developed by the authors (Weighted Stress Function method [14]).

The remaining of this paper is structured as follows. In section II, the different multi-criteria decision making methods considered in this work are presented, while the proposed measure is described in detail in section III. Experimental results are presented and discussed in section IV. The paper concludes with some directions for future work.

\section{MULTI-CRITERIA DECISION MAKING METHODS}

A significant number of multi-objective optimization methods are available in the literature. Solving an optimization problem consists of finding the Pareto-optimal solution that best satisfies the DM preferences. Multiobjective optimization problems are often solved by scalarization, i.e., by converting the original multiobjective formulation of the problem into a (or a family of) singleobjective optimization problem(s) by means of some realvalued function of the objectives, the scalarizing function. Such a scalarizing function may also depend on certain parameters, in order to allow it to incorporate preference information provided by the DM or, at least, to allow the DM to explore different regions of the Pareto frontier.

Four different scalarizing functions are described in this section. The first three are well-known classical approaches, whereas the fourth one has been proposed recently.

\section{A. Weighted Sum (WS)}

The weighted sum is perhaps the simplest and the most commonly used scalarizing function. The idea is to associate a weighting coefficient to each objective function, and to minimize the corresponding weighted sum of the objectives. Thus, the multiple objectives $\left(f_{i}\right)$ to be minimized are combined into a single objective $(F)$, as follows:

$$
\text { Minimize } \quad F(X)=\sum_{i=1}^{M} w_{i} f_{i}(X)
$$

where $x$ is a solution in the feasible decision variable space and $w_{i}$ is the $i^{\text {th }}$ component of a weighted vector such that $0 \leq w_{i} \leq 1$ and $w_{1}+w_{2}+\ldots+w_{M}=1$.

\section{B. Weighted Metrics}

The aim of the Weighted Metrics approach is to minimize the distance between solutions, represented in criterion space, and the ideal criterion vector $\left(z^{*}\right)$, which is the vector composed of the optimal objective values of each individual objective function separately. If the ideal criterion vector belongs to the space of admissible solutions, then the best solution will be the ideal solution, i.e. a solution that minimizes all of the objective functions simultaneously. However, this ideal vector will only belong to the admissible region when the criteria are not conflicting. With this method, a problem with $M$ objectives is changed into the following single objective problem:

$\operatorname{Minimize}\left(\sum_{i=1}^{M} w_{i}\left|f_{i}(X)-z_{i}^{*}\right|^{p}\right)^{1 / p}$

Subject to $X \in S, \quad 1 \leq p<\infty$

where $S$ is the feasible decision variable space, $w_{i}$ is the $i^{\text {th }}$ component of the normalized weight vector and $z^{*}{ }_{i}$ is the $i^{\text {th }}$ component of the ideal criterion vector. Commonly used values of $p$ are 1,2 and $\infty$, corresponding to the Manhattan, Euclidean and Tchebycheff distances, respectively. When $p=\infty$, equation 2 may also be written as:

$$
\begin{aligned}
& \text { Minimize } \max _{i=1, \ldots, M}\left\lfloor w_{i}\left|f_{i}(X)-z_{i}^{*}\right|\right]_{(3)} \\
& \text { Subject to } \quad X \in S
\end{aligned}
$$

This method is known as the Weighted Tchebycheff Metric (WTM) approach.

\section{Goal Programming (GP)}

In goal programming, the DM specifies aspiration levels $\left(Z_{i}\right)$ for the objective functions $\left(f_{i}\right)$, the goal being to meet these aspiration levels. Different approaches can be used to accomplish this, such as weighted, lexicographic and minmax goal programming [4].

In general, aspiration levels may or may not be attainable, so the aim is to minimize the differences (denoted as $\delta_{i}$ ) between the objective function values and the aspiration levels. If $\bar{Z}_{i}$ belongs to the feasible objective space, $\delta_{i}$ may be non-positive.

In the minimization case, a suitable formulation may be stated as follows:

$$
\begin{gathered}
\text { Minimize } \max _{i=1, \ldots, M}\left[f_{i}(X)-\bar{Z}_{i}\right] \\
\text { Subject to } \quad X \in S
\end{gathered}
$$

where $S$ is the feasible variable space, and $M$ is the number of objectives.

\section{Weighted Stress Function (WSF)}

This method is essentially a modification of the WTM approach where a non-linear function of each objective is used instead of the weights. The specific non-linear function used was constructed so as to mimic the stress-strain behaviour of thermoplastic vulcanizates [14].

The method will be explained with the aid of Fig. 1, where a Pareto frontier for an optimization problem with two criteria to be maximized, $f_{1}$ and $f_{2}$, is shown. For each solution belonging to the Pareto frontier, two stresses, $\sigma_{w l}$ and $\sigma_{w 2}$, are defined and associated with the corresponding criteria, $f_{1}$ and $f_{2}$ ( $w_{1}$ and $w_{2}$ are the weights assigned to each criterion). These stresses are proportional to the weighted distance between criteria $i$ and the $i^{\text {th }}$ component of the ideal 
objective vector.

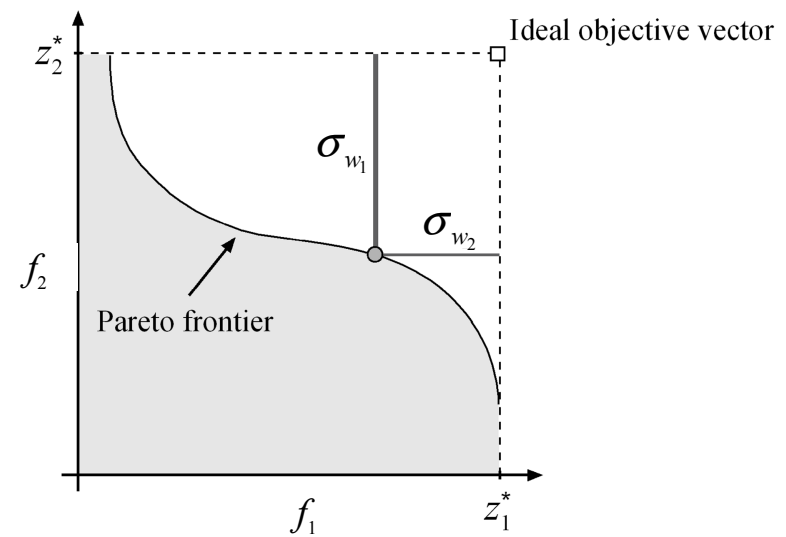

Fig. 1. Stresses associated with an optimal solution for an optimization problem with two criteria.

Since the two criteria are conflicting, the solution of the problem cannot be the ideal objective vector, and the stresses associated with the best solution for this problem will not be null. So, the best solution will be the one where the maximum stress associated to each criterion has the minimum possible value. This means that, for the best solution, the two stresses $\sigma_{w 1}$ and $\sigma_{w 2}$ will balance each other out, and, if the Pareto frontier is not discontinuous, the best vector for the problem will have the same stress associated to each criterion.

Formally, the scalarized problem may be stated as follows:

$$
\begin{aligned}
\text { Minimize } S(X) & =\max _{i=1, \ldots, m}\left\{\sigma_{w_{i}}\left(f_{i}(X)\right)\right\}(5) \\
\text { Subject to } \quad X & \in S
\end{aligned}
$$

where the weighted stress function associated to the weight $w_{i}$ is defined as:

$\sigma_{w_{i}}(x)=\left\{\begin{array}{lr}\frac{w_{i}}{2} \tan \left(-\frac{\pi}{\psi\left(w_{i}\right)}\left(x-w_{i}\right)\right)+\xi\left(w_{i}\right), & x \leq w_{i} \\ -\frac{\xi\left(w_{i}\right)}{\tan \left(\frac{\pi}{\varphi\left(w_{i}\right)}\left(w_{i}-1\right)\right)} \tan \left(-\frac{\pi}{\varphi\left(w_{i}\right)}\left(x-w_{i}\right)\right)+\xi\left(w_{i}\right), & x>w_{i}\end{array}\right.$

where,

$$
\begin{aligned}
& \varphi\left(w_{i}\right)=\frac{3}{4}\left(1-w_{i}\right)^{2}+2\left(1-w_{i}\right)+\delta_{1}(7) \\
& \psi\left(w_{i}\right)=\varphi\left(w_{i}\right)+4 w_{i}-2(8) \\
& \xi\left(w_{i}\right)=-\frac{1}{\tan \left(-\frac{\pi}{2+2 \delta_{2}}\right)} \tan \left(\frac{\pi}{1+\delta_{2}}\left(w_{i}-\frac{1}{2}\right)\right)+1
\end{aligned}
$$

This definition considers that the criteria are normalized, i.e., $f_{i} \in[0 ; 1]$. Consequently, the ideal vector has coordinates
$(1,1)$.

Fig. 2 represents the stress function $\left(\sigma_{w i}\right)$ and the geometric interpretation of functions $\varphi, \psi$ and $\xi$. For a given value of $w_{i}, \xi\left(w_{i}\right)$ is equal to $\sigma_{w i}\left(f_{i}\right)$ only when $f_{i}$ is equal to $w_{i}$ (since $f_{i}$ and $w_{i}$ are normalized, ranging in the interval $[0,1]$ ). Simultaneously, the distance between asymptote $\mathrm{A}_{1}$ and $w_{i}$ is $\psi\left(w_{i}\right) / 2$ and the distance between asymptote $\mathrm{A}_{2}$ and $w_{i}$ is $\varphi\left(w_{i}\right) / 2$. When $w_{i}$ and $f_{i}$ are near 0 or 1 , functions $\psi\left(w_{i}\right)$ and $\varphi\left(w_{i}\right)$ cannot be null in order to avoid that, in these cases, $\sigma_{w i}\left(f_{i}\right)$ tends to infinity. To allow the weight to range in the interval [0,1], a small value $\left(\delta_{l}\right)$ must be added to $\psi\left(w_{i}\right)$ and $\varphi\left(w_{i}\right)$ functions, without changing the shape of the $\sigma_{w i}\left(f_{i}\right)$ function considerably. Finally, function $\xi\left(w_{i}\right)$ must be as constant as possible in all $w_{i}$ domain, except for $w_{i}$ values near the interval limits (i.e., for values of $w_{i}$ near to 0 and 1 ). This is accomplished using the parameter $\delta_{2}$ used in 9. Various values of $\delta_{1}$ and $\delta_{2}$ were tested and suitable values

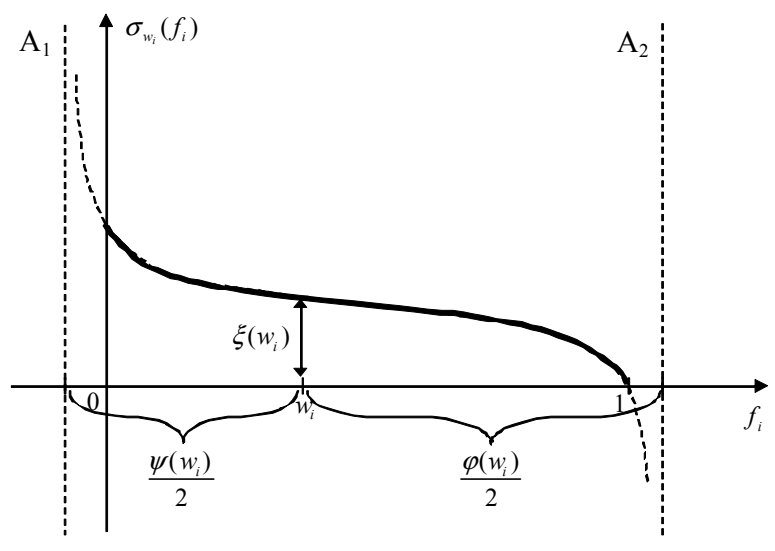

Fig. 2. Stress function for a specific weight.

were found to be $\delta_{1}=0.0001$ and $\delta_{2}=0.00001$. More details about this method can be found elsewhere [14].

\section{PERformance MEASURE}

All of the methods presented in the last section include parameters which the DM can use to explore different regions of the Pareto frontier. In general, the final solutions found will depend on the given parameter settings, but it is also desirable that this dependence is as direct as possible, so that the DM may use those parameters to express actual preferences. Although the properties of the relation between scalarizing function parameters and solutions has been the object of experimental studies before [15], the authors are unaware of any theoretical measure of the quality of such a mapping. 
In order to support the development of a suitable measure, one may argue that a good parameter-solution mapping should be such that:

1. All Pareto-optimal solutions correspond to some setting of the parameters

2. A uniform sampling of the parameter space leads to a good discrete approximation of the Pareto frontier

Therefore, it should be possible to use measures of the quality of discrete Pareto-set approximations [16], such as the hypervolume measure, to assess the quality of the parameter-solution relation induced by a specific scalarizing function on a given multiobjective optimization problem.

Since, in the biobjective case, the Pareto frontier is at most one-dimensional, a single parameter should suffice to enable a scalarizing function to generate all possible Paretooptimal solutions in that case. Denote that single parameter by $w$ and assume that it may take values in a finite-length interval $[a, b]$. Consider also a sequence of parameter values $w_{1}, w_{2}, \ldots, w_{k} \in[a, b]$, such that $w_{i+1}-w_{i}$ is constant for all $i$, and the corresponding sequences of objective values $f_{1,1}, f_{1,2}, \ldots$, $f_{l, k}$ and $f_{2,1}, f_{2,2}, \ldots, f_{2, k}$. Then, the area $A_{i}$ of the region which is dominated by the objective vector $\left(f_{1, i}, f_{2, i}\right)$ associated with parameter $w_{i}$, and which is not dominated by any other objective vector is

$$
A_{i}=\frac{f_{1, i+1}-f_{1, i}}{w_{i+1}-w_{i}} \times \frac{f_{2, i-1}-f_{2, i}}{w_{i+1}-w_{i}}
$$

as illustrated in Fig.3. One possible interpretation of a good parameter-solution mapping is one where all values of $A_{i}$ are approximately the same, for a family of Pareto fronts. By letting $k \rightarrow \infty$, both $w_{i+1}-w_{i}$ and $w_{l}-w_{i-1}$ will tend to zero, and 11 will lead to

$$
A(w)=\left|\frac{d f_{1}}{d w}\right| \times\left|\frac{d f_{2}}{d w}\right|(11)
$$

In principle, it should also be possible to extend this formulation to the many-objective case.

\section{RESULTS AND DiscUSSION}

\section{A. Test Problems}

The performance of the last three methods presented in section II will be assessed using the measure defined above. The five Test Problems (TP) described below, each of a different type and with distinctive Pareto frontier characteristics, will be used in this study. For each TP, the number $(L)$ of decision variables $(x)$, the corresponding ranges of variation, the number of criteria $(M)$, the corresponding expressions and the optimization direction (minimization or maximization) are given.

TP 1: $x \in[-2 ; 6]$; Minimize; $\mathrm{L}=1 ; \mathrm{M}=2$.

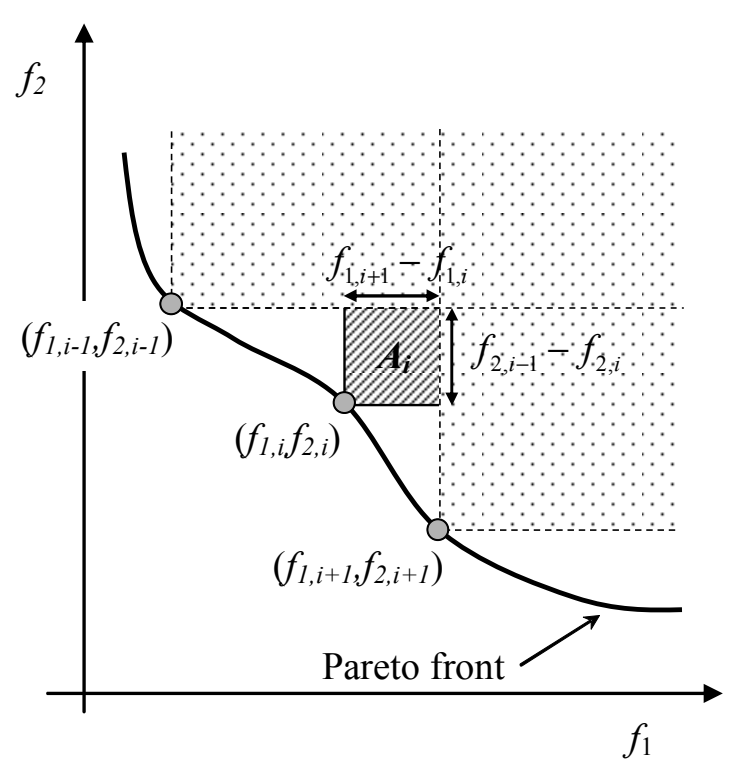

Fig. 3. Geometrical interpretation of the area $A_{i}$

$f_{1}(x)=x^{2}$

$f_{2}(x)=e^{|x|-5}+(6 / 5) \cos (2 x)-2,7 x+1$

TP 2: $x \in[-1 ; 1]$; Maximize; $\mathrm{L}=1 ; \mathrm{M}=2$.

$f_{1}(x)=-x^{2}+1$

$f_{2}(x)=\arctan (4 x)$

TP 3: $x \in[0 ; 1]$; Maximize; $\mathrm{L}=1 ; \mathrm{M}=2$.

$f_{1}(x)=x$

$f_{2}(x)=\frac{1}{40 x}$

TP 4 (ZDT1): $x_{i} \in[0 ; 1]$; Minimize; $\mathrm{L}=30 ; \mathrm{M}=2 ;[17]$.

$f_{1}\left(x_{1}\right)=x_{1}$

$f_{2}\left(x_{2}, \cdots, x_{L}\right)=g(x) \times\left(1-\sqrt{f_{1}\left(x_{1}\right) / g(x)}\right)$

with, $g(x)=1+9 \frac{\sum_{l=2}^{L} x_{l}}{L-1}$

TP 5 (ZDT2): $x_{i} \in[0 ; 1]$; Minimize; $\mathrm{L}=30 ; \mathrm{M}=2$; [17].

$f_{1}\left(x_{1}\right)=x_{1}$

$f_{2}\left(x_{2}, \cdots, x_{L}\right)=g(x) \times\left(1-\left(f_{1}\left(x_{1}\right) / g(x)\right)^{2}\right)$

with, $g(x)=1+9 \frac{\sum_{l=2}^{L} x_{l}}{L-1}$

\section{B. Results and Discussions}

In order to apply the methodology proposed, the objective functions were normalized (using the equation: $\left(f-f^{\min }\right) /\left(f^{\max }\right.$ $\left.f^{\mathrm{min}}\right)$ ) and thus they range between 0 and 1 .

Parameters $w_{1}$ and $w_{2}$ of the WTM and WSF methods 
were mapped onto a single parameter $w \in[-1,1]$, through the expression $w=w_{1}-w_{2}$, and similarly for the goal programming aspiration levels $\bar{Z}_{1}$ and $\bar{Z}_{2}$. Since the objective functions were normalized, aspiration levels were also normalized such that $\bar{Z}_{1}+\bar{Z}_{2}=1$, and $w=\bar{Z}_{1^{-}} \bar{Z}_{2}$.

A sequence of 200 distinct, equality spaced, values of the parameter $w$ covering the interval $[-1,1]$ was generated, and the Pareto optimal solution corresponding to each of those values was numerically determined for each scalarizing function considered. Then, the values of the function $A(w)$ were approximated using finite differences, and plotted.

Fig. 4 to 8 show the plots for the three methods under study, for the five test problems defined above. It can be seen that the values of $A(w)$ associated with WSF method and the GP formulation tend to vary less across the parameter range than in the case of the WTM method. On test problems 1, 2 and 4, WSF appears to be superior to the other methods, because $A(w)$ is more constant.

\section{CONCLUSIONS}

A measure allowing the comparison of the uniformity of the results obtained for MOOP has been proposed.

This measure was used to compare three different decision making methods for multi-objective optimization using five different test problems.

This comparison shown that the WSF method has the best performance when compared with the WTM and GP methods, i.e., the WSF has a better correspondence between the preferences of the DM and the solution obtained by the decision method.

\section{REFERENCES}

[1] C. M. Fonseca and P. J. Fleming, "Multiobjective optimization and multiple constraint handling with evolutionary algorithms-Part I: A unified formulation". IEEE Transactions on Systems, Man and Cybernetics, vol. 28, pp. 26-37, 1998.

[2] C. M. Fonseca and P. J. Fleming, "Genetic algorithms for multiobjective optimization: Formulation, discussion and generalization", in Proc. 5th International Conf. on Genetic Algorithms, San Mateo,1994, pp.141-153.

[3] K. Deb, Multi-objective Optimization using Evolutionary Algorithms. Chichester: Wiley, 2001.

[4] K. M. Miettinen, Nonlinear Multiobjective Optimization. Boston: Kluwer, 1999.

[5] C. L. Hwang and A. S. Masud, Multiple Objective Decision Making Methods and Applications. Berlin: Springer-Verlag 1979.

[6] H. Trautmann and J. Mehnen, "Preference-Based Pareto-Optimization in Certain and Noisy Environments", Engineering Optimization, Vol. 41, pp. 23-38, 2009.

[7] Y. Aksoy, T. W. Butler and E. D. Minor, "Comparative studies in interactive multiple objective mathematical programming", European Journal of Operational Research, vol. 89, pp. 408-422, 1996.

[8] G. R. Reeves and J. J. Gonzalez., "A comparison of two interactive MCDM procedures", European Journal of Operational Research, vol. 41, pp. 203-209, 1989.

[9] B. F. Hobbs and P. M., Meier, "Multicriteria methods for resource planning: An experimental comparison", IEEE Transactions on Power Systems, vol. 9, pp. 1811-1817, November 1994,.

[10] J. S. Dyer and R. K. Sarin, "Measurable multiattribute value functions", Operations Research, vol. 27, pp. 810-822, July 1979.

[11] B. Malakooti and A. Ravindran, "Experiments with an interactive paired comparison simplex method for MOLP problems", Operations Research, vol5, pp. 575-597, 1985.
[12] R. L. Keeney and H. Raiffa, Decisions With Multiple Objectives: Preferences and Value Tradeoffs. New York: Wiley, 1976.

[13] B. F. Hobbs, "What Can We Learn From Experiments in Multiobjective Decision Analysis?", IEEE Transactions on Systems, Man and Cybernetics, vol. 16, pp. 384-394, May 1986.

[14] J. C. Ferreira, C. M. Fonseca and A. Gaspar-Cunha, "Methodology to select solutions for multi-objective optimization problems: Weight stress function method", Applied Intelligence, to appear.

[15] K. Miettinen and M. M. Mäkelä, "On scalarizing functions in multiobjective optimization", OR Spectrum, vol. 24 ,pp. 193-213, 2002.

[16] E. Zitzler, L. Thiele, M. Laumanns, C. M. Fonseca and V. G. Fonseca, "Performance Assessment of multiobjective optimizers: An analysis and review", IEEE Transactions on Evolutionary Computation, vol.7, pp. 117-132, April 2003.

[17] K. Deb, A. Pratap, S. Agrawal, and T. Meyarivan, "A fast and elitist multi-objective genetic algorithm: NSGA-II", IEEE Trans. on Evol. Comp., vol. 6, pp. 182-197, 2002.

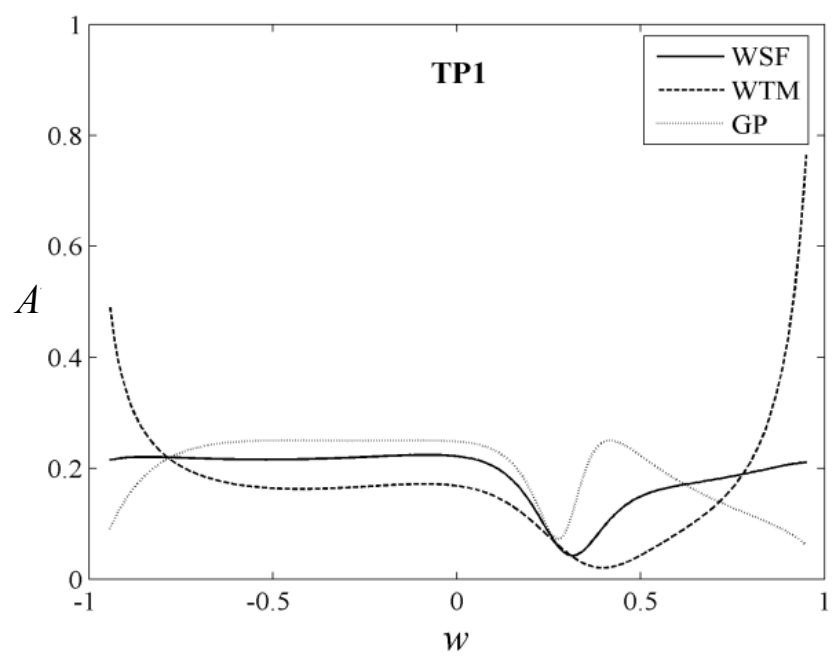

Fig. 4. Consistent results for WSF, WTM and GP methods for TP1.

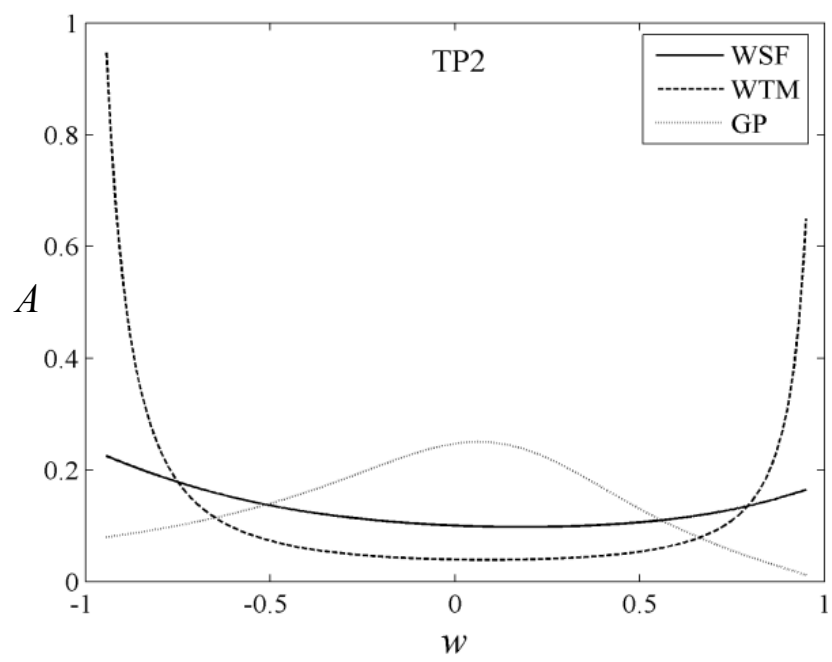

Fig. 5. Consistent results for WSF, WTM and GP methods for TP2. 


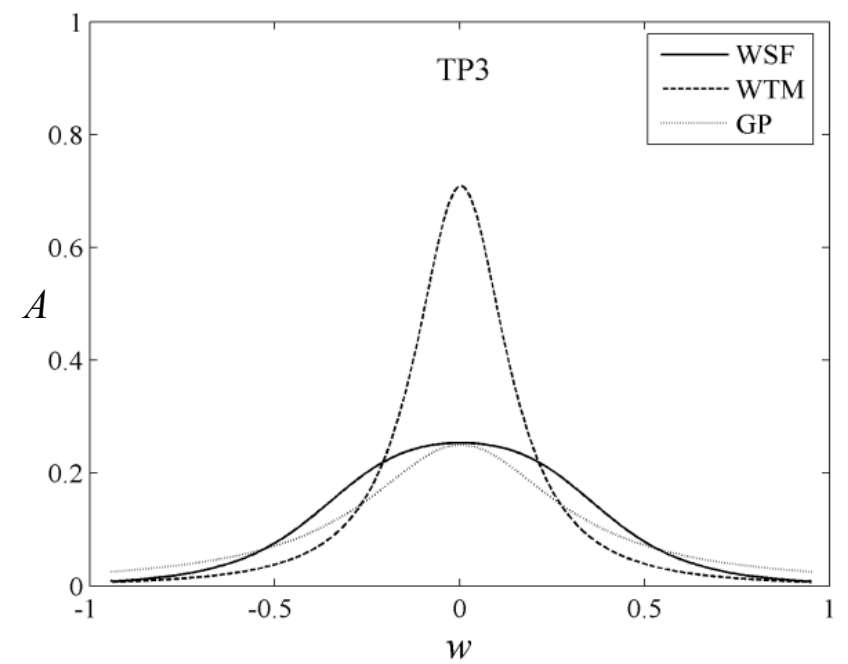

Fig. 6. Consistent results for WSF, WTM and GP methods for TP3.

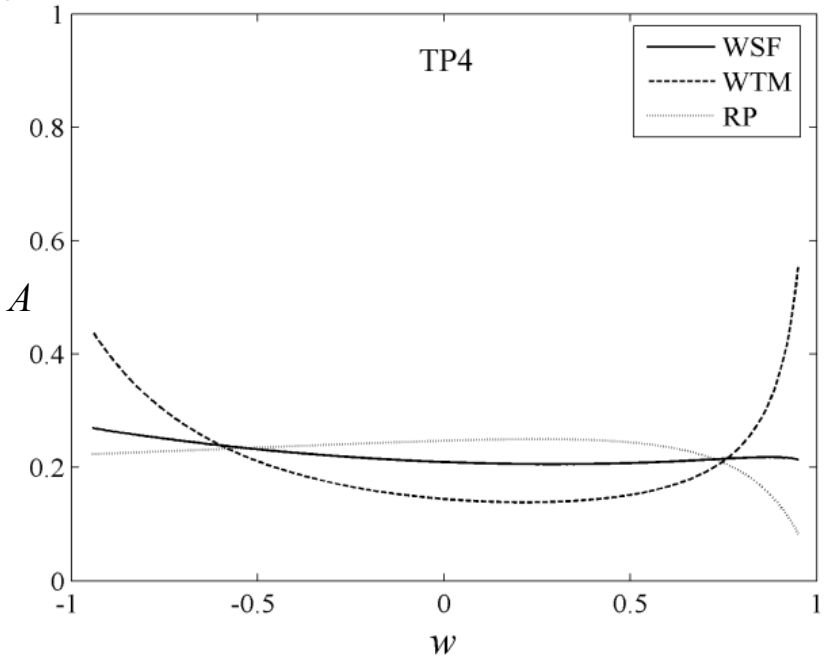

Fig. 7. Consistent results for WSF, WTM and GP methods for TP4.

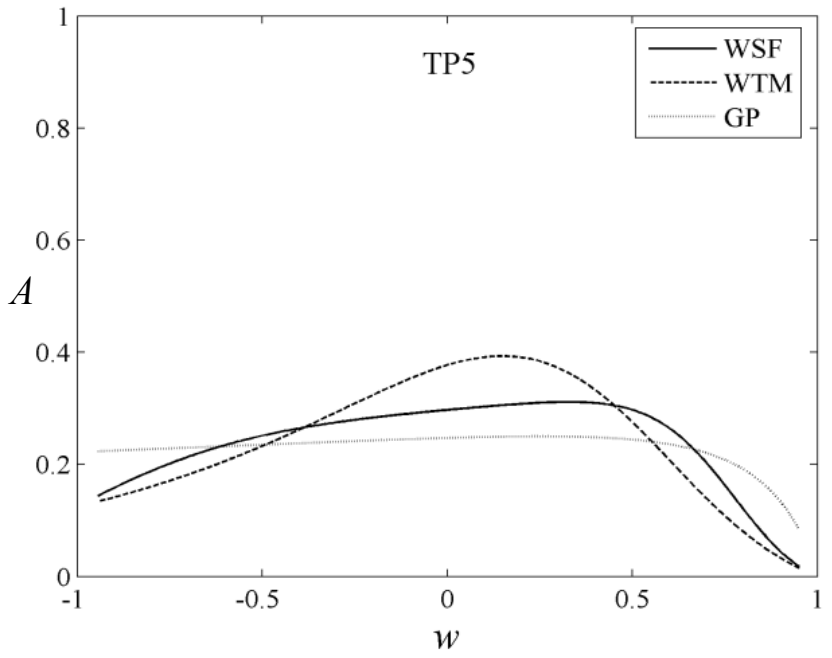

Fig. 8. Consistent results for WSF, WTM and GP methods for TP5. 